\title{
Metabolic syndrome-related features in controlled and resistant hypertensive subjects
}

\author{
Arthur Santa Catharina*, Rodrigo Modolo, Alessandra Ritter, Andréa Sabbatini, Nathália Corrêa, Veridiana \\ Brunelli, Nayara Fraccari, Aurélio Almeida, Heitor Moreno, Ana Paula de Faria.
}

\begin{abstract}
Objective: The purpose of this study was to evaluate the prevalence of metabolic syndrome (MetS) and the clinical features associated with it in resistant and mild to moderate hypertensives. Methods: This cross-sectional study included 236 patients, (i) 129 mild to moderate hypertensive patients and (ii) 107 patients with resistant hypertension (RHTN). We determined blood pressure measurements and adipokines levels. Target organ damages such as microalbuminuria (MA), cardiac hypertrophy and arterial stiffness were also assessed. Results: We found a prevalence of $73 \%$ in resistant and $60 \%$ in mild-to-moderate hypertensive patients. The patients with MetS showed a higher prevalence of MA $\geq 30 \mathrm{mg} \cdot \mathrm{g}^{-1}$ compared to their counterparts $(20 \% \mathrm{vs}$. $4 \%$ ). Adiponectin levels were significantly lower in patients with MetS (5.30 vs. $7.50 \mu \mathrm{g} \cdot \mathrm{mL}^{-1}$ ), while leptin demonstrated to be increased in those patients, compared to the subjects without MetS (21.0 vs. $\left.15.7 \mathrm{ng} \cdot \mathrm{mL}^{-1}\right)$. Finally, in a multiple regression analysis $\mathrm{MA}(\mathrm{OR}=8.51 ; \mathrm{p}=0.01)$, leptin/adiponectin ratio (LAR) $(\mathrm{OR}=4.13 ; \mathrm{p}=0.01)$ and $\mathrm{RHTN}(\mathrm{OR}=3.75 ; \mathrm{p}=0.03)$ were independently associated with the presence of MetS, apart from potential confounders. Conclusions: Our findings suggest that the metabolic derangements present in MetS tend to develop early signs of end-organ damage with hormonal changes in hypertensive patients. Indeed, LAR may be useful as a reliable biomarker for identifying those who are at risk for developing MetS.
\end{abstract}

\section{Key words: \\ Hypertension, Adipokines, Obesity.}

\section{Introduction}

High blood pressure and resistance to antihypertensive therapy are associated with various anthropometric and metabolic abnormalities ${ }^{1}$ including abdominal obesity, elevated triglycerides, reduced high-density lipoprotein cholesterol, glucose intolerance and insulin resistance. The mechanisms of obesity-related hypertension include, among others, inflammatory adipokines ${ }^{2}$ and overactivation of the renin-angiotensin system, which have demonstrated a significant role in the pathogenesis of metabolic syndrome (MetS) and resistant hypertension $(\mathrm{RH})$. The purpose of this study was to evaluate the prevalence of MetS and the clinical features associated with it in resistant and mild to moderate hypertensives.

\section{Results and Discussion}

This cross-sectional study included 236 patients, (i) 129 mild to moderate hypertensive patients and (ii) 107 patients with $\mathrm{RH}$. We determined blood pressure measurements and adipokines levels. Target organ damages such as microalbuminuria (MA), cardiac hypertrophy and arterial stiffness were also assessed.

We found a MetS prevalence of $66 \%$ in all hypertensive population. Neither office and ambulatory BP levels nor the proportion of patients with uncontrolled office BP $(>140 / 90 \mathrm{mHg})$ were different between groups. The patients with MetS showed a higher prevalence of MA compared to their counterparts. Additionally, adiponectin levels were significantly lower in patients with MetS, while leptin demonstrated to be increased in those patients, compared to the subjects without MetS.

Finally, the multiple logistic regression revealed that $M A$, leptin/adiponectin ratio and resistance to antihypertensive treatment were independently associated with the presence of MetS (Table 1)
Table 1 - Multiple logistic regression for the presence of MetS*

\begin{tabular}{|c|c|c|c|}
\hline & Odds ratio & $95 \% \mathrm{Cl}$ & p-value \\
\hline LAR $>3.7$ & 4.13 & $1.38-12.34$ & $0.01^{*}$ \\
\hline HR (bpm) & 0.97 & $0.92-1.03$ & 0.39 \\
\hline $\mathrm{MA}>30\left(\mathrm{mg} \cdot \mathrm{g}^{-1}\right)$ & 8.51 & $1.53-47.14$ & $0.01^{*}$ \\
\hline hs-CRP (mg.dL-1) & 2.92 & $0.83-10.19$ & 0.09 \\
\hline RHTN & 3.75 & $1.09-12.92$ & $0.03^{*}$ \\
\hline
\end{tabular}

\section{Conclusions}

Our findings suggest that the metabolic derangements present in MetS tend to develop early signs of end-organ damage with hormonal changes in hypertensive patients. Indeed, LAR may be useful as a reliable biomarker for identifying those who are at risk for developing MetS.

\section{Acknowledgement}

This study was supported by the São Paulo Research Foundation (FAPESP) and National Council for Scientific and Technological Development (CNPq), Brazil.

\section{References}

1. Lopes HF, et al. Visceral adiposisty syndrome. Diabetol Metab Syndr. 2016; 8 : 40 .

2. de Faria AP, et al. Hypoadiponectinemia and aldosterone excess are associated with lack of blood pressure control in subjects with resistant hypertension. Hypertens Res. 2013; 36:1067-7. 